\title{
Bounded-Degree Graphs have Arbitrarily Large Queue-Number
}

\author{
David R. Wood" \\ Departament de Matemàtica Aplicada II \\ Universitat Politècnica de Catalunya \\ Barcelona, Spain \\ david.woodeupc.es
}

received August 14, 2006, revised August 28, 2007, accepted December 21, 2007.

It is proved that there exist graphs of bounded degree with arbitrarily large queue-number. In particular, for all $\Delta \geq 3$ and for all sufficiently large $n$, there is a simple $\Delta$-regular $n$-vertex graph with queue-number at least $c \sqrt{\Delta} n^{1 / 2-1 / \Delta}$ for some absolute constant $c$.

Keywords: graph, queue layout, queue-number, track layout, track-number

Mathematics Subject Classification: 05C62 (graph representations), 05C30 (enumeration of graphs and maps)

\section{Introduction}

We consider graphs possibly with loops but with no parallel edges. A graph without loops is simple. Let $G$ be a graph with vertex set $V(G)$ and edge set $E(G)$. If $S \subseteq E(G)$ then $G[S]$ denotes the spanning subgraph of $G$ with edge set $S$. We say $G$ is ordered if $V(G)=\{1,2, \ldots,|V(G)|\}$. Let $G$ be an ordered graph. Let $\ell(e)$ and $r(e)$ denote the endpoints of each edge $e \in E(G)$ such that $\ell(e) \leq r(e)$. Two edges $e$ and $f$ are nested and $f$ is nested inside $e$ if $\ell(e)<\ell(f)$ and $r(f)<r(e)$. An ordered graph is a queue if no two edges are nested. Observe that the left and right endpoints of the edges in a queue are in first-in-first-out order-hence the name 'queue'. An ordered graph $G$ is a $k$-queue if there is a partition $\left\{E_{1}, E_{2}, \ldots, E_{k}\right\}$ of $E(G)$ such that each $G\left[E_{i}\right]$ is a queue.

Let $G$ be an (unordered) graph. A $k$-queue layout of $G$ is a $k$-queue that is isomorphic to $G$. The queue-number of $G$ is the minimum integer $k$ such that $G$ has a $k$-queue layout. Queue layouts and queuenumber were introduced by Heath et al. [15, 16] in 1992, and have applications in sorting permutations [12, 17, 23, 25, 29], parallel process scheduling [3], matrix computations [24], and graph drawing [4, 6]. Other aspects of queue layouts have been studied in [7, 8, 10, 14, 26, 27, 30].

\footnotetext{
${ }^{\dagger}$ Research supported by a Marie Curie Fellowship from the European Commission under contract MEIF-CT-2006-023865, and by the projects MEC MTM2006-01267 and DURSI 2005SGR00692.

1365-8050 @ 2008 Discrete Mathematics and Theoretical Computer Science (DMTCS), Nancy, France
} 
Prior to this work it was unknown whether graphs of bounded degree have bounded queue-number. The main contribution of this note is to prove that there exist graphs of bounded degree with arbitrarily large queue-number.

Theorem 1 For all $\Delta \geq 3$ and for all sufficiently large $n>n(\Delta)$, there is a simple $\Delta$-regular $n$-vertex graph with queue-number at least $c \sqrt{\Delta} n^{1 / 2-1 / \Delta}$ for some absolute constant $c$.

The best known upper bound on the queue-number of a graph with maximum degree $\Delta$ is e $(\Delta n / 2)^{1 / 2}$ due to Dujmović and Wood [8] (where e is the base of the natural logarithm). Observe that for large $\Delta$, the lower bound in Theorem 1 tends toward this upper bound. Although for specific values of $\Delta$ a gap remains. For example, for $\Delta=3$ we have an existential lower bound of $\Omega\left(n^{1 / 6}\right)$ and a universal upper bound of $\mathcal{O}\left(n^{1 / 2}\right)$.

Closely related to a queue layout is the notion of a track layout. Informally speaking, a track layout of a graph consists of a proper vertex colouring, and a total order of each colour class, such that between each pair of colour classes, no two edges cross (with respect to the orders of the colour classes that contain the endpoints of the edges). The track-number of a graph $G$, denoted by $\operatorname{tn}(G)$, is the minimum number of colours in a track layout of $G$; see [4-7, 9,-11, 13]. Dujmović et al. [6] proved that qn $(G) \leq$ $\operatorname{tn}(G)-1$. Thus a lower bound on the queue-number also provides a lower bound on the track-number ${ }^{(i)}$ In particular, Theorem 1 implies:

Theorem 2 For all $\Delta \geq 3$ and for all sufficiently large $n>n(\Delta)$, there is a simple $\Delta$-regular $n$-vertex graph with track-number at least $c \sqrt{\Delta} n^{1 / 2-1 / \Delta}$ for some absolute constant $c$.

Note that there is also a $\mathcal{O}\left(n^{1 / 2}\right)$ upper bound on the track-number of graphs with bounded degree. The best result is $\operatorname{tn}(G) \leq 5 \Delta(G) \sqrt{2 n}$, which follows from the result by Dujmović and Wood [11] that $\operatorname{tn}(G) \leq 5 d \sqrt{2 n}$ for every $d$-degenerate $e^{(i i)}$ graph $G$. Thus for large $\Delta$, the lower bound in Theorem 2 tends toward this upper bound.

\section{Proof of Theorem 1}

The proof of Theorem 1 is modelled on a similar proof by Barát et al. [1]. Basically, we show that there are more $\Delta$-regular graphs than graphs with bounded queue-number. The following lower bound on the number of $\Delta$-regular graphs is a corollary of more precise bounds due to Bender and Canfield [2], Wormald [31], and McKay [22]; see [1].

Lemma $1(\mid \mathbf{2}, 22$, 31|) For all integers $\Delta \geq 1$ and for sufficiently large $n \geq n(\Delta)$, the number of labelled simple $\Delta$-regular $n$-vertex graphs is at least

$$
\left(\frac{n}{3 \Delta}\right)^{\Delta n / 2}
$$

It remains to count the graphs with bounded queue-number. We will need the following lemma, which was previously known only for loopless graphs.

Lemma 2 ([י8]) Every $n$-vertex queue has at most $2 n-1$ edges.

(i) A converse result in fact holds. Dujmović et al. 7] proved that track-number is bounded by a function of queue-number. In particular, $\operatorname{tn}(G) \leq 4 \mathrm{qn}(G) \cdot 4^{\mathrm{qn}(G)(2 \mathrm{qn}(G)-1)(4 \mathrm{qn}(G)-1)}$ for every graph $G$.

(ii) A graph $G$ is $d$-degenerate if every subgraph of $G$ has a vertex of degree at most $d$. 
Proof: If $v+w=x+y$ for two distinct edges $v w$ and $x y$, then $v w$ and $x y$ are nested. No two edges are nested in a queue. The result follows since $2 \leq v+w \leq 2 n$.

Let $g(n)$ be the number of queues on $n$ vertices. For our purposes it suffices to show that $g(n) \leq$ $c^{n}$. While such a bound can be concluded from the work of Klazar [19], who described a generating function for $g(n)$ with rough asymptotic analysis, we include a different and simpler proof for the sake of completeness. It is similar to a proof of a more general result by Klazar [20]; also see [21, 28] for other related and more general results.

Lemma $3 g(n) \leq c^{n}$ for some absolute constant $c$.

Proof: Say $G$ is an $n$-vertex queue. Let $G^{\prime}$ be an ordered $2 n$-vertex graph obtained by the following doubling operation. For every edge $v w$ of $G$, add to $G^{\prime}$ a non-empty set of edges between $\{2 v-1,2 v\}$ and $\{2 w-1,2 w\}$, no pair of which are nested. If $v \neq w$ then there are 11 possible ways to do this, as illustrated in Figure 1 1 and if $v=w$ then there are 7 possible ways to do this, as illustrated in Figure 2 . Now $G$ has at most $2 n-1$ edges by Lemma 2 . Thus at most $11^{2 n-1}$ queues on $2 n$ vertices can be obtained from $G$ by doubling. On the other hand, every $2 n$-vertex queue can be obtained from some $n$-vertex queue by doubling. To see this, merge every second pair of vertices, introduce a loop between merged vertices that are adjacent, and replace any resulting parallel edges by a single edge. No two edges are nested in the obtained graph. Hence $g(2 n) \leq 11^{2 n-1} \cdot g(n)$. It follows that $g(n) \leq 11^{2 n}$.

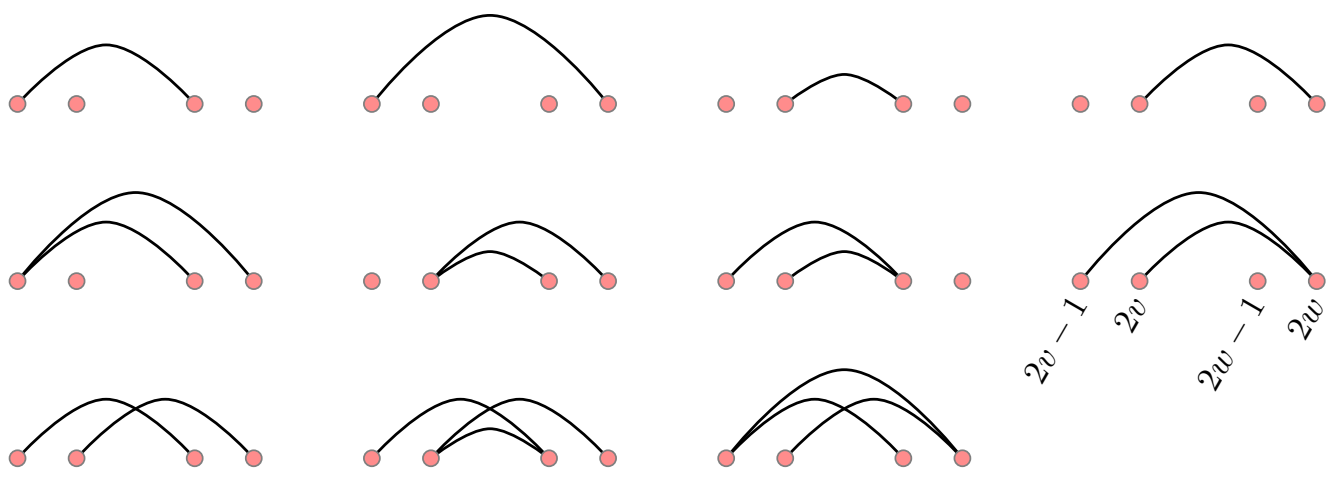

Fig. 1: The 11 possible ways to add a non-empty set of non-nested edges between $\{2 v-1,2 v\}$ and $\{2 w-1,2 w\}$.

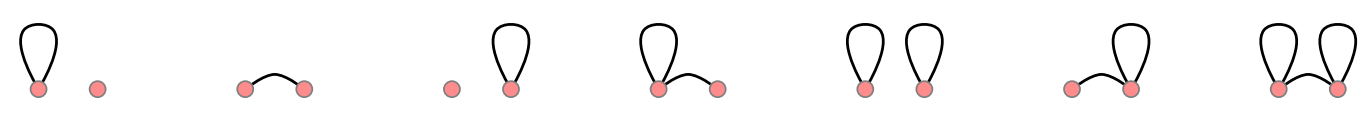

Fig. 2: The 7 possible ways to add a non-empty set of edges on $2 v-1$ and $2 v$.

Lemma 3 implies:

Corollary 1 The number of $k$-queues on $n$ vertices is at most $c^{k n}$ for some absolute constant $c$. 
It is easily seen that Lemma 1 and Corollary 1 imply a lower bound of $c(\Delta / 2-1) \log n$ on the queuenumber of some $\Delta$-regular $n$-vertex graph. To improve this logarithmic bound to polynomial, we now give a more precise analysis of the number of $k$-queues that also accounts for the number of edges in the graph. Let $g(n, m)$ be the number of queues on $n$ vertices and $m$ edges.

\section{Lemma 4}

$$
g(n, m) \leq \begin{cases}\left(\begin{array}{c}
n \\
2 m
\end{array}\right) \cdot c^{2 m} & , \text { if } m \leq \frac{n}{2} \\
c^{n} & , \text { if } m>\frac{n}{2}\end{cases}
$$

for some absolute constant $c$.

Proof: By Lemma 3 we have the upper bound of $c^{n}$ regardless of $m$. Suppose that $m \leq \frac{n}{2}$. An $m$-edge graph has at most $2 m$ vertices of non-zero degree. Thus every $n$-vertex $m$-edge queue is obtained by first choosing a set $S$ of $2 m$ vertices, and then choosing a queue with $|S|$ vertices. The result follows.

Let $g(n, m, k)$ be the number of $k$-queues on $n$ vertices and $m$ edges.

Lemma 5 For all integers $k$ such that $\frac{2 m}{n} \leq k \leq m$,

$$
g(n, m, k) \leq\left(\frac{c k n}{m}\right)^{2 m}
$$

for some absolute constant $c$.

Proof: Fix non-negative integers $m_{1} \leq m_{2} \leq \cdots \leq m_{k}$ such that $\sum_{i} m_{i}=m$. Let $g\left(n ; m_{1}, m_{2}, \ldots, m_{k}\right)$ be the number of $k$-queues $G$ on $n$ vertices such that there is a partition $\left\{E_{1}, E_{2}, \ldots, E_{k}\right\}$ of $E(G)$, and each $G\left[E_{i}\right]$ is a queue with $\left|E_{i}\right|=m_{i}$. Then

$$
g\left(n ; m_{1}, m_{2}, \ldots, m_{k}\right) \leq \prod_{i=1}^{k} g\left(n, m_{i}\right) .
$$

Now $m_{1} \leq \frac{n}{2}$, as otherwise $m>\frac{k n}{2} \geq m$. Let $j$ be the maximum index such that $m_{j} \leq \frac{n}{2}$. By Lemma 4 .

$$
g\left(n ; m_{1}, m_{2}, \ldots, m_{k}\right) \leq\left(\prod_{i=1}^{j}\left(\begin{array}{c}
n \\
2 m_{i}
\end{array}\right) c^{2 m_{i}}\right)\left(c^{n}\right)^{k-j} .
$$

Now $\sum_{i=1}^{j} m_{i} \leq m-\frac{1}{2}(k-j) n$. Thus

$$
\begin{aligned}
g\left(n ; m_{1}, m_{2}, \ldots, m_{k}\right) & \leq\left(\prod_{i=1}^{j}\left(\begin{array}{c}
n \\
2 m_{i}
\end{array}\right)\right)\left(c^{2 m-(k-j) n}\right)\left(c^{(k-j) n}\right) \\
& \leq c^{2 m} \prod_{i=1}^{k}\left(\begin{array}{c}
n \\
2 m_{i}
\end{array}\right) .
\end{aligned}
$$


We can suppose that $k$ divides $2 m$. It follows (see [1]) that

$$
g\left(n ; m_{1}, m_{2}, \ldots, m_{k}\right) \leq c^{2 m}\left(\begin{array}{c}
n \\
2 m / k
\end{array}\right)^{k} .
$$

It is well known [18, Proposition 1.3] that $\left(\begin{array}{l}n \\ t\end{array}\right)<(\mathrm{e} n / t)^{t}$. Thus with $t=2 m / k$ we have

$$
g\left(n ; m_{1}, m_{2}, \ldots, m_{k}\right)<\left(\frac{c \mathrm{e} k n}{2 m}\right)^{2 m}
$$

Clearly

$$
g(n, m, k) \leq \sum_{m_{1}, \ldots, m_{k}} g\left(n ; m_{1}, m_{2}, \ldots, m_{k}\right)
$$

where the sum is taken over all non-negative integers $m_{1} \leq m_{2} \leq \cdots \leq m_{k}$ such that $\sum_{i} m_{i}=m$. The number of such partitions [18, Proposition 1.4] is at most

$$
\left(\begin{array}{c}
k+m-1 \\
m
\end{array}\right)<\left(\begin{array}{c}
2 m \\
m
\end{array}\right)<2^{2 m} .
$$

Hence

$$
g(n, m, k) \leq 2^{2 m}\left(\frac{c \mathrm{e} k n}{2 m}\right)^{2 m}
$$

Every ordered graph on $n$ vertices is isomorphic to at most $n$ ! labelled graphs on $n$ vertices. Thus Lemma 5 has the following corollary.

Corollary 2 For all integers $k$ such that $\frac{2 m}{n} \leq k \leq m$, the number of labelled $n$-vertex m-edge graphs with queue-number at most $k$ is at most

$$
\left(\frac{c k n}{m}\right)^{2 m} n !
$$

for some absolute constant $c$.

Proof of Theorem 1; Let $k$ be the minimum integer such that every simple $\Delta$-regular graph with $n$ vertices has queue-number at most $k$. Thus the number of labelled simple $\Delta$-regular graphs on $n$ vertices is at most the number of labelled graphs with $n$ vertices, $\frac{1}{2} \Delta n$ edges, and queue-number at most $k$. By Lemma 1 and Corollary 2 .

$$
\left(\frac{n}{3 \Delta}\right)^{\Delta n / 2} \leq\left(\frac{c k}{\Delta}\right)^{\Delta n} n ! \leq\left(\frac{c k}{\Delta}\right)^{\Delta n} n^{n}
$$

Hence $k \geq \sqrt{\Delta} n^{1 / 2-1 / \Delta} /(\sqrt{3} c)$. 


\section{Acknowledgements}

Thanks to Martin Klazar who found an error in an early draft of this paper.

\section{References}

[1] JÁnOS BARÁt, JiŘí MATOUŠEK, AND DAVID R. WOOD. Bounded-degree graphs have arbitrarily large geometric thickness. Electron. J. Combin., 13(1):R3, 2006.

[2] Edward A. Bender And E. Rodney CANFIELD. The asymptotic number of labeled graphs with given degree sequences. J. Combin. Theory Ser. A, 24:296-307, 1978.

[3] Sandeep N. Bhatt, Fan R. K. Chung, F. Thomson Leighton, and Arnold L. RosenBERG. Scheduling tree-dags using FIFO queues: A control-memory trade-off. J. Parallel Distrib. Comput., 33:55-68, 1996.

[4] Emilio Di Giacomo, Giuseppe Liotta, and Henk Meijer. Computing straight-line 3D grid drawings of graphs in linear volume. Comput. Geom., 32(1):26-58, 2005.

[5] Emilio Di Giacomo, Giuseppe Liotta, Henk Meijer, and Stephen K. Wismath. Volume requirements of 3D upward drawings. In Patrick Healy and Nikola S. Nikolov, eds., Proc. 13th International Symp. on Graph Drawing (GD '05), vol. 3843 of Lecture Notes in Comput. Sci., pp. 101-110. Springer, 2006.

[6] Vida Dujmović, PAT Morin, And David R. Wood. Layout of graphs with bounded tree-width. SIAM J. Comput., 34(3):553-579, 2005.

[7] Vida Dujmović, Attila Pór, And David R. Wood. Track layouts of graphs. Discrete Math. Theor. Comput. Sci., 6(2):497-522, 2004.

[8] Vida Dujmović And David R. Wood. On linear layouts of graphs. Discrete Math. Theor. Comput. Sci., 6(2):339-358, 2004.

[9] Vida Dujmović And David R. Wood. Three-dimensional grid drawings with sub-quadratic volume. In JÁnos PACH, ed., Towards a Theory of Geometric Graphs, vol. 342 of Contemporary Mathematics, pp. 55-66. Amer. Math. Soc., 2004.

[10] Vida Dujmović And DAVID R. WoOd. Stacks, queues and tracks: Layouts of graph subdivisions. Discrete Math. Theor. Comput. Sci., 7:155-202, 2005.

[11] Vida Dujmović And David R. Wood. Three-dimensional upward grid drawings of graphs. Order, 23:1-20, 2006.

[12] Shimon Even And A. ItAi. Queues, stacks, and graphs. In Zvi KoHAVi And Azaria PAZ, eds., Proc. International Symp. on Theory of Machines and Computations, pp. 71-86. Academic Press, 1971.

[13] Stefan Felsner, Giussepe Liotta, and Stephen K. Wismath. Straight-line drawings on restricted integer grids in two and three dimensions. J. Graph Algorithms Appl., 7(4):363-398, 2003. 
[14] Joseph L. Ganley. Stack and queue layouts of Halin graphs. 1995. http://www.ganley.org/pubs/Halin.pdf.

[15] Lenwood S. Heath, F. Thomson Leighton, and Arnold L. Rosenberg. Comparing queues and stacks as mechanisms for laying out graphs. SIAM J. Discrete Math., 5(3):398-412, 1992.

[16] Lenwood S. Heath and Arnold L. Rosenberg. Laying out graphs using queues. SiAM J. Comput., 21(5):927-958, 1992.

[17] Atsumi ImamiYa AND AKIHIRo NozAKi. Generating and sorting permutations using restricteddeques. Information Processing in Japan, 17:80-86, 1977.

[18] STASys Jukna. Extremal combinatorics: with applications in computer science. Texts in Theoretical Computer Science. Springer, 2001.

[19] Martin Klazar. Counting pattern-free set partitions. II. Noncrossing and other hypergraphs. Electron. J. Combin., 7:34, 2000.

[20] Martin Klazar. The Füredi-Hajnal conjecture implies the Stanley-Wilf conjecture. In Proc. 12th International Conference on Formal Power Series and Algebraic Combinatorics (FPSAC '00), pp. 250-255. Springer, 2000.

[21] AdAm Marcus ANd GÁbor TARdos. Excluded permutation matrices and the Stanley-Wilf conjecture. J. Combin. Theory Ser. A, 107(1):153-160, 2004.

[22] Brendan D. McKay. Asymptotics for symmetric 0-1 matrices with prescribed row sums. Ars Combin., 19(A):15-25, 1985.

[23] Edward T. Ordman and William Schmitt. Permutations using stacks and queues. In Proc. 24th Southeastern International Conf. on Combinatorics, Graph Theory, and Computing, vol. 96 of Congr. Numer., pp. 57-64. 1993.

[24] Sriram V. Pemmaraju. Exploring the Powers of Stacks and Queues via Graph Layouts. Ph.D. thesis, Virginia Polytechnic Institute and State University, U.S.A., 1992.

[25] Vaughan R. Pratt. Computing permutations with double-ended queues. Parallel stacks and parallel queues. In Proc. 5th Annual ACM Symp. on Theory of Computing (STOC '73), pp. 268-277. ACM, 1973.

[26] S. Rengarajan and C. E. Veni Madhavan. Stack and queue number of 2-trees. In Ding-Zhu Du And Ming LI, eds., Proc. 1st Annual International Conf. on Computing and Combinatorics (COCOON '95), vol. 959 of Lecture Notes in Comput. Sci., pp. 203-212. Springer, 1995.

[27] Farhad Shahrokhi and Weiping Shi. On crossing sets, disjoint sets, and pagenumber. $J$. Algorithms, 34(1):40-53, 2000.

[28] Gábor Tardos. On 0-1 matrices and small excluded submatrices. J. Combin. Theory Ser. A, 111(2):266-288, 2005. 
[29] Robert E. Tarjan. Sorting using networks of queues and stacks. J. Assoc. Comput. Mach., 19:341-346, 1972.

[30] David R. Wood. Queue layouts of graph products and powers. Discrete Math. Theor. Comput. Sci., 7(1):255-268, 2005.

[31] Nicholas Wormald. Some problems in the enumeration of labelled graphs. Ph.D. thesis, Newcastle-upon-Tyne, United Kingdom, 1978. 